\title{
LA IMPORTANCIA DE LA PLANEACIÓN FINANCIERA EN LA ELABORACIÓN DE LOS PLANES DE NEGOCIO Y SU IMPACTO EN EL DESARROLLO EMPRESARIAL*
}

\author{
JAIME ANDRÉS CORREA GARCÍA*, LEIDY JOHANA RAMÍREZ BEDOYA* \\ \& CARLOS EDUARDO CASTAÑO RÍOS \\ UNIVERSIDAD DE ANTIOQUIA
}

Recibido/Received/Recebido: 27/02/2010 - Aceptado/ Accepted/Aprovado: 26/05/2010

\begin{abstract}
Resumen
Con el creciente estímulo a los programas de emprendimiento empresarial por las administraciones públicas, universidades, entidades de fomento y público en general; los planes de negocio han cobrado especial importancia en el ámbito de la gestión empresarial. En este sentido, este artículo tiene como objetivo identificar la importancia estratégica de la planeación financiera para la creación de empresas sostenibles en el tiempo. Para lograr lo anterior, se realizó una revisión bibliográfica de diversos temas relacionados con la elaboración de planes de negocio y la continuidad de las empresas, y se complementó a través de la experiencia en la evaluación de dichos planes. De este modo, se identificaron siete falencias comunes en la planeación financiera por parte de los emprendedores. Se concluye que la planeación financiera se constituye en la base para la continuidad de las nuevas empresas que se crean a partir de planes de negocio.
\end{abstract}

Palabras clave: plan de negocio, planeación financiera, creación de empresas, emprendedor

\section{IMPORTANCE OF FINANCIAL PLANNING IN BUSINESS PLAN ELABORATION AND ITS IMPACT ON ENTERPRISE DEVELOPMENT}

\begin{abstract}
With the growing stimulation to entrepreneurship programs by public administrations, universities, development entities and in general; business plans have increased its importance in the enterprise management scope. In this sense, this article has the purpose of identifying strategic importance of financial planning to crate sustainable companies along time. In order to achieve this, a biblio-
\end{abstract}

Artículo de reflexión, derivado del proyecto de investigación "Modelo de gestión financiera integral para PYMES: prospectiva en escenarios de competitividad global" financiado por el Comité de Investigaciones de la Universidad de Antioquia - CODI. Proyecto llevado a cabo por el Grupo de Investigaciones y Consultorías en Ciencias Contables.

* Contador Público. Especialista en Finanzas, Preparación y Evaluación de Proyectos de la Universidad de Antioquia, Magister en Ingeniería Financiera, Universidad Nacional de Colombia. Coordinador de la línea de investigación en Gestión Financiera y Gestión de MIPYMES del Grupo de Investigaciones y Consultorías en Ciencias Contables - GICCO. Docente de tiempo completo del Departamento de Ciencias Contables de la Universidad de Antioquia. Profesor y Consultor en el Parque del Emprendimiento de la Universidad de Antioquia y la Alcaldía de Medellín. Correo electrónico: jaimecorrea@udea.edu.co.

*** Contadora Pública de la Universidad de Antioquia. Integrante del Grupo de Investigaciones y Consultorías en Ciencias Contables - GICCO de la Universidad de Antioquia. Correo electrónico: leidyramirez18@yahoo.es

Contador Público Universidad de Antioquia. Integrante del Grupo de Investigaciones y Consultorías en Ciencias Contables - GIC$\mathrm{CO}$ de la Universidad de Antioquia. Docente de tiempo completo del Departamento de Ciencias Contables de la Universidad de Antioquia. Correo electrónico: carloscontaduria@gmail.com 
graphic review of various topics related to business plan elaboration and company continuity was made, and it was complemented with the evaluation experience of those plans. Thus, seven common failures were identified in financial planning by entrepreneurs. The conclusion is that financial planning is the base for continuity of new companies created from a business plan.

Keywords: business plan, financial planning, business creation, entrepreneurship.

\title{
A IMPORTÂNCIA DO PLANEJAMENTO FINANCEIRO NA PREPARAÇÃO DOS PLANOS DE NEGÓCIO E SEU EFEITO NO DESENVOLVIMENTO EMPRESARIAL
}

\author{
Resummo
}

\begin{abstract}
Com o incentivo cada vez maior aos programas de empreendedorismo por parte das administrações públicas, universidades, agências de desenvolvimento e público geral, os planos de negócios têm uma importância especial no domínio da gestão empresarial. Portanto, o objetivo deste artigo é identificar a importância estratégica do planejamento financeiro para a criação de firmas sustentáveis. Em conseqüência, nós revisamos a literatura relacionada com a preparação de planos de negócios e a continuidade das firmas, e a complementamos com a experiência na avaliação de tais planos. Assim, se identificaram sete fraquezas comuns aos empresários no planejamento financeiro. Nós concluímos que o planejamento financeiro é a base para a continuidade de novas empresas criadas a partir de planos de negócios.
\end{abstract}

Palavras chave: Plano de negócio, planejamento financeiro, criação de firmas, empreendedor

Correa, J., Ramírez, L. \& Castaño, C. (2010). La importancia de la planeación financiera en la elaboración de los planes de negocio y su impacto en el desarrollo empresarial. En: Revista de la Facultad de Ciencias Económicas de la Universidad Militar Nueva Granada. rev.fac.cienc.econ, XVIII (1)

JEL: M13.

\section{Introducción}

La creación de empresas a partir de planes de negocios está tomando cada vez más fuerza en Colombia. Desde esta perspectiva, se deben revisar algunos elementos que hacen parte de este proceso de planeación empresarial, específicamente, en lo referente a la planeación financiera y su incidencia en la construcción del denominado estudio financiero o plan financiero que hace parte del plan de negocio.

Este artículo tiene como principal objetivo identificar la importancia estratégica de la planeación financiera para la creación de empresas sostenibles en el tiempo. El trabajo desarrolla en sus primeros apartes la metodología para la construcción de este artículo, los conceptos generales de plan de negocio, sus componentes y la articulación de los mismos con el plan financiero, con el fin de ingresar en la temática de la planeación financiera, la cual influye significativamente sobre el futuro de los proyectos empresariales en su paso de proyecto a empresa sostenible. Luego, se exponen algunas falencias comunes que han sido identificadas en la asesoría de diversos planes de negocios tomando como referente algunos ejemplos sacados del plan financiero de proyectos empresariales reales. Por último, se presentan las consideraciones finales al respecto de la temática trabajada en el artículo, con el fin de generar un ambiente de discusión en torno a la planeación financiera en los procesos de creación de empresas sostenibles a partir de planes de negocios. 


\section{Metodología}

Para el desarrollo de este artículo de reflexión, el equipo de investigación realizó la revisión de diversos textos académicos acerca de la construcción de planes de negocios, evaluación y preparación de proyectos, continuidad empresarial y creación de valor. La revisión documental comprendió principalmente los siguientes autores: Varela (2001), Sapag \& Sapag (2003), Black \& Tarquin (2006), Meléndez (2005), Correa, Jaramillo, Ramírez \& Castaño (2008), Correa \& Jaramillo (2007), Frixione (2004), Mejía (2005), Ramírez \& Vanegas (2008), Sánchez (2006), Castaño (2009), García (1999), Castaño \& Ramírez (2009), Ramírez (2006) y Soto \& Dolan (2004). Cada uno de los textos revisados permitió evaluar distintos aspectos sobre las situaciones que se pueden presentar en la construcción de los planes de negocio en sus distintos componentes, así como las implicaciones de esa planeación y futura sostenibilidad de las nuevas empresas que entran a formar parte del mercado.

Asimismo, la experiencia de los autores en el campo del apoyo para la elaboración y evaluación de planes de negocio en distintos eventos, facilitó la reflexión sobre siete (7) falencias o errores comunes que se evidencian en la planeación financiera de los planes de negocio al momento del proceso evaluativo, ya que muchos de los proyectos evaluados durante los últimos años arrojan como resultado las tendencias que se analizan en este artículo y que pueden servir como guía para que los futuros emprendedores no caigan en estos errores comunes por causa de una planeación financiera inadecuada para sus organizaciones. De este modo, se ilustra a los lectores con algunos ejemplos tomados de varios planes de negocio, en los cuales se lograron evidenciar las llamadas falencias comunes, sin pretender agotar con ello la existencia de más falencias en la planeación financiera en los planes de negocio.

\section{Consideraciones sobre planes de negocio}

El proceso de creación de empresas está supeditado a un sinnúmero de variables que influyen en la estructuración y puesta en marcha de las mismas. Va- riables de tipo técnico, administrativo, legal, financiero y económico, por citar algunas, constituyen el punto de partida para la creación de empresas sostenibles en el tiempo, por lo que es indispensable su análisis concienzudo en el proceso de planeación empresarial. Así, al impregnarle un carácter estratégico a la forma de desarrollar este tipo de proyectos, no sólo desde el punto de vista operativo sino también financiero, es posible lograr un equilibrio en todos los niveles de la futura organización, de forma que se puedan afrontar los posibles cambios $y$ tendencias del entorno con mejores condiciones.

En este sentido, resulta imperante contar con herramientas administrativas y de gestión que permitan consolidar los procesos de creación de empresas y poner en funcionamiento los proyectos empresariales. Tal es el caso del denominado plan de negocio, el cual puede ser definido como un documento escrito que permite evaluar la viabilidad comercial, técnica, jurídica, administrativa y financiera de un proyecto empresarial y la definición de mecanismos para su puesta en marcha. En éste se intentan plasmar los objetivos, métodos, estrategias, y actos que la empresa desarrollará en el futuro, a fin de identificar, describir y analizar su oportunidad, crecimiento y proyección.

Conceptualmente, y en términos un poco más técnicos, es posible encontrar diversas definiciones en torno al significado de "Plan de Negocios". En primer lugar, se puede traer a colación la definición planteada por el profesor Rodrigo Varela, en la cual manifiesta que:

El plan de negocios es un proceso de darle al negocio una identidad, una vida propia. Es un procedimiento para enunciar de forma clara y precisa los propósito, las ideas, los conceptos, las formas operativas, los resultados, y en resumen la visión del empresario sobre el proyecto. Es un mecanismo de proyectar la empresa en el futuro, de prever dificultades y de identificar posibles soluciones a las coyunturas que pudiesen presentarse (Varela, 2001,160).

La anterior definición permite evidenciar aspectos clave en un plan de negocio como la concreción de 
las ideas de negocio a través de la planeación, lo cual permite generar mecanismos de choque para futuros problemas cuando la empresa se encuentre en marcha. Por su parte, el profesor Humberto Meléndez plantea que un plan de negocio es "un documento que identifica, describe y analiza la oportunidad de cristalizar una idea de inversión, examina la viabilidad en cuanto al mercadeo del bien o servicio, parte técnica y financiera de la misma, desarrolla todos los procedimientos y estrategias necesarias para convertir la citada oportunidad de negocio en un proyecto concreto" (Meléndez, 2005,9).

En el portal de internet del Ministerio de Comercio, Industria y Turismo de Colombia, se encuentra que un plan de negocios es un "Bloque de información, expresada en un documento, que tiene que ser comprendido por propios y extraños y que evidencia un pensamiento estratégico de la empresa y sus negocios, con obligaciones y resultados cuantificables previstos para un período de tiempo determinado" (Ministerio de Comercio, Industria y Turismo, 2006,1).

Por su parte, el Programa Gestión Tecnológica de la Universidad de Antioquia presenta la siguiente definición sobre plan de negocios:

"Se entiende como el esfuerzo en la recolección y orden de las ideas, en la dirección de abarcar todas las variables que influirán sobre el proyecto, con el objetivo de interrelacionarlas y encontrar la combinación óptima de las mismas en el estudio; éste pasa a constituirse en una forma de visualizar el futuro, es una herramienta potente de planeación que conduce a la disminución del riesgo al considerar elementos críticos y permitir adelantarse a ellos. El Plan de Negocios es importante porque resume la oportunidad del negocio, define y articula cómo el equipo gestor espera alcanzar y ejecutar la oportunidad identificada e interesa a posibles inversores para obtener recursos y capacidades necesarias para iniciar la empresa. Es la formulación de un Plan de Negocios el camino que nos permite asignar eficientemente los recursos, ya que su 'poder de previsión' y su flexibilidad para visionar diferentes escenarios, permite de manera cuantitativa y cualitativa dimensionar las ventajas o desventajas de las posibles decisiones" (Programa Gestión Tecnológica, Universidad de Antioquia, 2008,2).

Resulta claro que la esencia de los planes de negocio radica en brindar al emprendedor una estructura más profunda acerca de su modelo de negocio. En forma más específica, el plan intenta dar respuesta a los siguientes interrogantes: qué, cómo, dónde, para qué, por qué y cuándo, con el fin de marcar la pauta para el accionar del emprendedor con relación a la puesta en marcha de su idea de negocio.

Entre los aspectos positivos a destacar al momento de realizar un plan de negocios se encuentra en primer lugar, siguiendo a Meléndez (2005), que es un documento de planeación, en el cual se consignan las estrategias y tácticas que permitirán llegar posteriormente a la creación de una nueva empresa. Además, teniendo en cuenta lo planteado por Varela (2001) sobre un plan de negocios, se establece que permite visualizar el negocio en el futuro e identificar nuevas oportunidades, de este modo, un plan consiste en utilizar información histórica y presente para decidir hoy lo que se va a realizar en el futuro, lo cual debe partir de una planificación adecuada que establezca objetivos y metas realistas, tanto a largo como a corto plazo para lo que resulta relevante tener clara una visión que proyecte el accionar del negocio en un futuro, pues esto implica un sentido de continuidad en éste.

Un plan de negocios también coadyuva en la toma de decisiones y por ello, cuando la idea de negocio se estructura correctamente a partir de un plan claramente delimitado, el emprendedor cuenta con información que le permite tomar acertadas decisiones para beneficio de su actividad empresarial. De las decisiones que se tomen en el presente, basadas en un plan concreto, se desprende el éxito del futuro. Adicionalmente, Varela (2001) expresa que "permite reducir los riesgos del proyecto, al tomar decisiones con más información y de mejor calidad" (162). Por otro lado, el plan facilita la conexión de la empresa con su entorno (clientes, proveedores, inversionistas, gobierno, aliados y competidores). El emprendedor debe tener claro que no 
operará como un ente aislado, sino que estará en permanente interrelación con diferentes grupos de interés que impactan, ya sea positiva o negativamente, su actividad. A partir del plan se realiza un estudio del ambiente externo de la empresa y de cómo se pueden aprovechar todas las oportunidades que le brinda el mercado abierto en el que se desenvuelve, pero igualmente sirve para detectar las amenazas que implican operar en el entorno capitalista actual.

Por último, es un punto de referencia para medir el desempeño de la empresa durante su implementación. Es claro que al estructurar el plan se tiene una aproximación a los resultados que posiblemente se obtendrán, ya que en el plan se hacen proyecciones financieras, de gestión, sociales y legales que permiten visualizar en el presente, el accionar futuro (Sapag \& Sapag, 2003).

\section{Componentes del plan de negocios}

Una vez abordada entonces la conceptualización sobre el Plan de Negocios, resulta necesario hacer alusión de manera sintética a los diferentes componentes que lo conforman, los cuales permiten estructurar el proyecto empresarial y su posterior puesta en marcha. Los componentes genéricos para la realización de un Plan de Negocios son: análisis $e$ investigación de mercados, estudio legal, estudio técnico, estudio administrativo, estudio de impactos sociales y ambientales y estudio financiero. A continuación se describe cada uno de los componentes mencionados:

\subsection{Análisis e investigación de mercado}

Es importante empezar la elaboración del plan de negocios, recopilando información acerca del comportamiento del mercado, ya que a partir de éste se pueden conocer cuáles son las empresas o negocios similares que prestan un servicio o producen un bien parecido al que se quiere dar a conocer y qué beneficios ofrecen. Además, también facilita determinar cuáles serán las necesidades que se pretenden satisfacer con el proyecto empresarial, cuáles son los posibles consumidores y qué es lo que realmente quieren, cuál es su capacidad de pago, qué es lo que efectivamente están dispuestos a comprar y a qué precios, cuáles son los productos o servicios sustitutos, entre otros. Varela, expresa que el análisis de mercado es "con gran frecuencia, el más difícil del proceso, y se convierte en la piedra angular de cuyos resultados se desprenden muchos otros análisis" (Varela, 2001,165).

Si bien no en todos los casos es imprescindible contratar una investigación de mercado, debe buscarse la información apoyándose en fuentes de diversa índole, como revistas y diarios especializados, cámaras de comercio, internet, consultores o personas que ya están en el mercado, encuestas, entrevistas, etc., las cuales pueden aportar información valiosa para el sustento del plan de negocios.

Por otra parte, resulta significativo que en esta etapa del plan de negocios, se defina un perfil del cliente potencial, se analicen las oportunidades, riesgos y estrategias de incursionar en el mercado, se examine como está conformado el mercado de los proveedores, de los competidores y de los distribuidores, para que así se pueda proporcionar una mirada global de la situación y de las perspectivas que se tendrán en el futuro.

En resumen, un estudio de mercados, es un informe que debe brindar información como demandas, ofertas, precios, estrategias comerciales y su costo, productos y servicios, ventajas competitivas, comparativas, absolutas y relativas de los diferentes negocios, entre otros, todo encaminado a orientar al inversionista a analizar factores preponderantes para poner en marcha el plan de negocio o la estrategia productiva.

\subsection{Estudio legal}

Para el desarrollo del plan de negocio, es necesario establecer un marco legal bien estructurado, que incorpore toda la reglamentación requerida para una adecuada implementación del mismo, con el fin de conocer cuáles son las normas que regulan en materia legal la actividad que se pretende desarrollar, desde su constitución y puesta en marcha, hasta la operación del proyecto en el momento en que se convierte en empresa. Dentro de los aspectos legales 
que hay que tener en cuenta al momento de definir el plan de negocio se encuentran: a) Definición de la forma jurídica de operación. b) Definición de los requisitos legales para la constitución. Se debe consultar el régimen legal para cada forma jurídica y en particular la elegida. c) Investigación de la existencia de algún tipo de régimen especial para el proyecto empresarial, por ejemplo para negocios ambientales, hoteleros, de salud o tecnológicos, generalmente existen disposiciones particulares que benefician la implementación de proyectos en estos campos.

Una buena definición legal es indispensable porque siempre se debe operar bajo un adecuado marco normativo, correspondiente a derechos y deberes de cada una de las partes involucradas en el proyecto, conforme a lo estipulado en la ley. Además, se tienen que analizar aspectos laborales, tributarios, políticos, sociales, ambientales, legales, administrativos, entre otros, ya que es importante considerar los distintos efectos que traerían estos al negocio, ya sean positivos o negativos.

\subsection{Estudio técnico}

Se determina la forma para lograr la elaboración y venta del producto o la prestación del servicio que se tiene previsto ofrecer, con la calidad, cantidad y costo requerido. Dentro de este estudio, se incluyen aspectos como el diseño, la tecnología, los procesos, los recursos tanto humanos como físicos, así como todas aquellas actividades necesarias para llevar a cabo el plan de negocio. Éste incluye: estudio de la capacidad, de la localización, de procesos y balances técnicos.

Así mismo, en este punto, se debe dar respuesta al "saber hacer", ¿qué hace la empresa?, al "cómo hacerlo", ¿cuáles son los macro y micro procesos que se desarrollan? y finalmente, al "qué se necesita”, ¿cuáles son los recursos requeridos para llevar a cabo la elaboración del producto o la prestación del servicio? Una vez respondidos tales interrogantes, se procede entonces a determinar los componentes técnicos necesarios para la operación del negocio tales como maquinaria y equipos, implementos y herramientas de oficina, estructura física, así como su ubicación y funcionamiento.
Es importante también determinar el diseño y estructura de los procesos administrativos y productivos del negocio, teniendo en cuenta aspectos de ingeniería, tales como remodelaciones, adecuaciones, transformaciones y mejoras (preoperativos), y la formulación de estrategias de puesta en marcha, funcionamiento y consolidación del mismo. Aquí es conveniente hacer una descripción detallada de todos aquellos recursos que se necesitan para desarrollar esta etapa técnica, iniciando con todo lo relacionado con el producto o servicio, hasta llegar a su forma de gestión y control. Es de mucha ayuda utilizar gráficos, flujogramas, matrices y demás estructuras que faciliten y mejoren la comprensión.

En este punto, la definición de los costos de producción o prestación de servicio es un elemento bastante importante, ya que define de alguna forma la capacidad competitiva a nivel de precios en el mercado frente a las otras empresas de competencia directa o indirecta. En este sentido, los profesores Ramírez \& Vanegas (2008) plantean algunas soluciones para la gestión de costos para este tipo de empresas nacientes.

\subsection{Estudio administrativo}

Se inicia con la elaboración de la carta organizacional donde se establece la estructura de la organización y el perfil de los cargos que tendrá la empresa, determinando el recurso humano de carácter administrativo necesario para la operación del negocio; así como algunos elementos complementarios en su administración y que estimulan el desarrollo de la actividad del negocio tales como: estrategias motivacionales, plan de capacitaciones e inducciones, recompensas e incentivos, entre otros. También se incluyen algunos requerimientos de bienes que serán utilizados por estas personas para su normal desempeño en la organización tales como muebles y equipos de oficina.

\subsection{Estudio de impactos ambientales y sociales (EIAS)}

Es importante tener presente que todo plan de negocios que se pretende instaurar, se encuentra inmer- 
so en una dinámica económica, social, ambiental y política, por lo que se requiere la determinación de los distintos impactos que se generan en torno a los mismos, ya que estos constituyen factores fundamentales en la sostenibilidad del proyecto como tal.

A nivel ambiental, resulta útil la identificación de cada una de las condiciones actuales y futuras del medio ambiente, dado que éstas constituyen un factor fundamental para el desarrollo sostenible del proyecto empresarial, puesto que las nuevas exigencias y prácticas administrativas enmarcan la gestión ambiental como un elemento primordial $e$ inherente a las actividades de operación de cada ente. Se habla entonces de Mitigar, Controlar, Corregir, Compensar y Prevenir los distintos impactos que se deriven de los procesos y actividades de producción y prestación de servicios.

A nivel social, es importante también determinar los impactos que sobre la comunidad generará el proyecto, ya que a fin de cuentas es a la sociedad a quien se pretende satisfacer con la ejecución del mismo y sobre quien se logrará aportar un beneficio social y económico.

\subsection{Estudio financiero}

Con este tipo de estudio, lo que se pretende es determinar cómo se va obtener el capital necesario para poner en marcha la empresa, cómo se permitirá el funcionamiento, cómo va a financiarse el crecimiento y desarrollo de los negocios y finalmente, qué beneficios va a generar la inversión realizada. En esta etapa se pretende medir la rentabilidad del negocio y si es viable desde el punto de vista financiero (Blank \& Tarquin, 2006 y Sapag \& Sapag, 2003). Es importante considerar conceptos claves en el lenguaje de las finanzas como Flujo de Caja del Proyecto (Flujo de Caja Libre Operacional - FCLO), Flujo de caja del Inversionista (FCI), Valor Presente Neto (VPN), Tasa Interna de Retorno (TIR), Período de Recuperación de la Inversión (PRI), Relación Beneficio/Costo ( $\mathrm{RBC}$ ), indicadores de liquidez, rentabilidad y endeudamiento, entre muchos otros, ya que es en esta etapa en donde se resume y se traduce a un lenguaje más cuantitativo los demás componentes, con el fin de determinar el presupuesto integral (cualitativo y cuantitativo) requerido para llevar a cabo el proyecto.

\section{Articulación del plan de negocios y el plan financiero}

Es muy importante destacar la fuerte vinculación que presentan todos los componentes antes mencionados con el plan de negocios, al constituir parte integral del mismo, aunque cada uno tiene una finalidad distinta y trabaja una temática diferente según el tipo de estudio que se pretende realizar, los resultados y análisis repercuten en el proyecto como tal. Así las cosas, la elaboración de un plan de negocios resulta siendo un proceso sistémico que requiere la aplicación de estudios previos de acuerdo a los componentes que lo conforman a fin de conocer la viabilidad del proyecto de creación de empresa, lo cual conduce a la generación de unos resultados que se reflejan de forma cuantitativa en el estudio financiero.

A continuación se muestran algunos ejemplos de los impactos generados por cada componente del plan de negocios sobre el plan financiero, lo que denota la consistencia que debe darse en todo el proceso de elaboración.

- Estudio legal: su impacto en lo financiero está determinado en los estudios previos - Inversión diferida- y en el establecimiento de obligaciones que afectarán los flujos de caja, principalmente los impuestos.

- Estudio de mercados: impacta lo financiero con la determinación de cantidades a vender y precios, los planes de mercadeo y capital de trabajo - cartera-.

- Estudio técnico: su impacto financiero es notorio, porque determina necesidades de inversión fija y en capital de trabajo - inventarios- así como flujos de costos.

- Estudio administrativo: impacta lo financiero con la estimación de los gastos de la estructura organizacional y el desarrollo de planes operativos.

- $\quad$ Estudio de impactos ambiental y salud (EIAS): impacta lo financiero en la medida que las con- 
Gráfico 1. Articulación del Plan de Negocios y el Plan Financiero

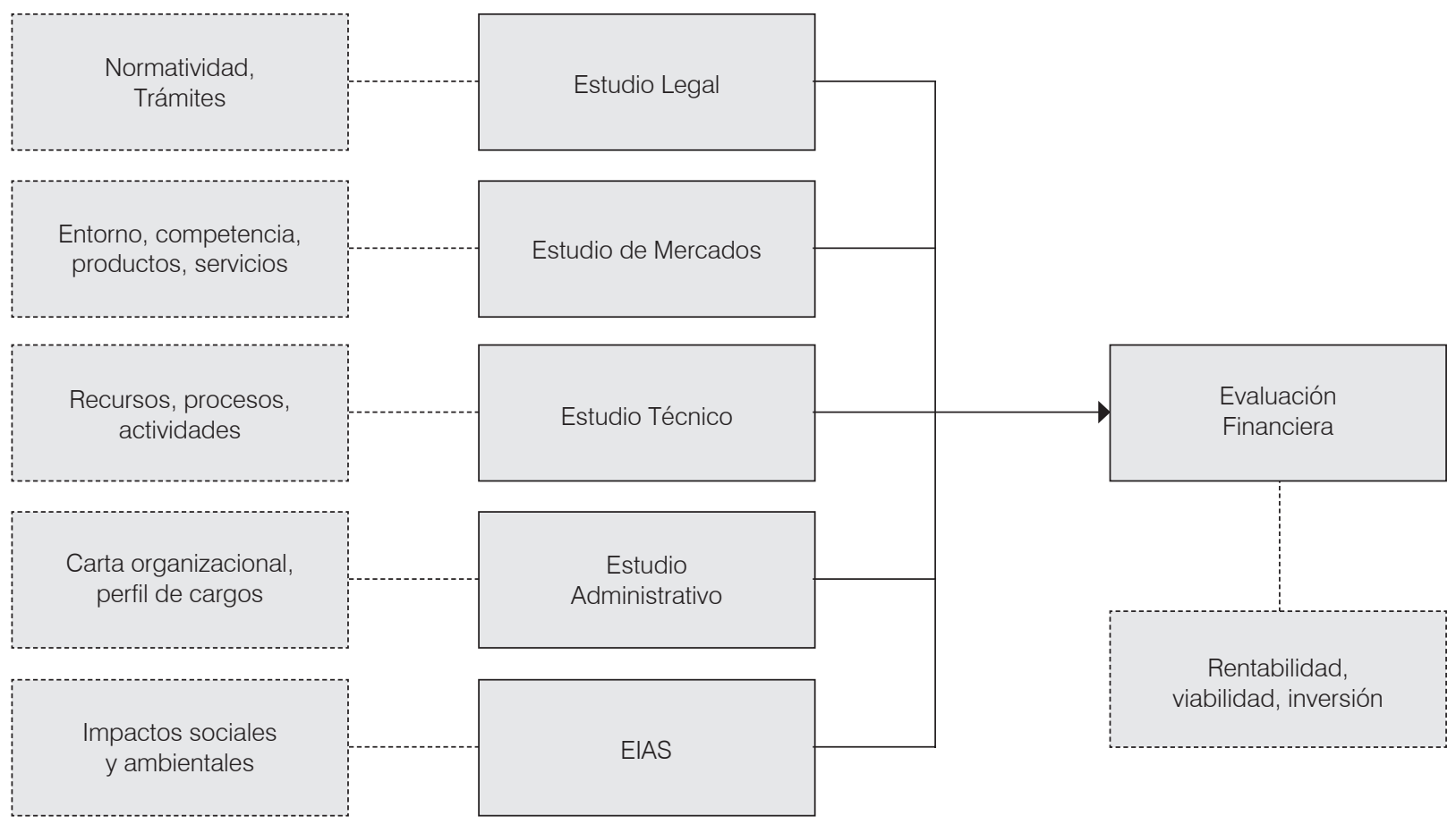

clusiones pueden llevar a la adopción de planes de contingencia, pago de contribuciones especiales, programas de promoción y prevención, etc.

\section{La planeación financiera}

Con el fin de llevar a cabo la realización de un buen plan de negocios, es conveniente tener en cuenta el proceso de planeación financiera, entendida como una herramienta empresarial que permite la visualización del proyecto empresarial bajo un enfoque global, teniendo en cuenta los diferentes escenarios en donde puede incursionar y los distintos factores que lo impactan. Dicho en otras palabras, este proceso, es el que permite traducir a términos cuantitativos (financieros) todas las políticas, prácticas y estrategias contempladas en el plan de negocios mediante la modelación financiera, la cual permite la realización de proyecciones y estimaciones financieras, imprimiéndole un carácter estratégico que apunta a la adecuada toma de decisiones. En términos conceptuales, es preciso citar una definición encontrada en torno a la planeación financiera:

“(...) entendido no sólo como un modelo de proyecciones financieras que arroja los Estados Financieros de resultados, balance y flujos de recursos con sus respectivos indicadores; sino también como un conjunto de actividades que se desarrollan principalmente en el nivel estratégico (tanto de negocio como funcional) y en menor grado, en el nivel operativo. Bajo estas consideraciones se puede decir que la planeación financiera es un "proceso en el cuál se transforma, en términos financieros, los planes estratégicos y operativos del negocio en un horizonte de tiempo determinado; suministrando información que soporta las

1 Elaboración de los autores. 
decisiones tanto estratégicas como financieras propiamente dichas", (...) "Un proceso integral y consciente de planeación financiera como herramienta empresarial, nos permite comprender y visualizar la estrategia global del negocio a la luz de los siguientes tres tipos de decisiones a nivel financiero: la de comprometer recursos (inversiones), la de estructura de capital (capital y/o préstamos requeridos, riesgo) y la de los dividendos para los accionistas" (Sánchez, 2006,155).

En este sentido, la planeación financiera es una herramienta de suma importancia en la gestión empresarial, ya que permite integrar de forma armónica cada uno de los componentes del plan de negocios traduciéndolos a términos, no sólo financieros- cuantitativos sino también cualitativos. De esta manera, es posible lograr una mayor comprensión del proyecto de creación de empresa, tener la capacidad de estimar las condiciones futuras, cuantificar y cualificar los riesgos asociados a la operación, evaluar el modo de operar y las estrategias contempladas, establecer ciertos escenarios, y lo más importante, contar con un fundamento estratégico para la creación de empresas sostenibles en el tiempo (Correa \& Jaramillo, 2007). Este proceso se debe realizar de manera transversal en los distintos estudios del plan de negocios, ya que la experiencia denota que el componente financiero es uno de los más débiles debido a la falta de formación de los equipos emprendedores en el tema, lo que lleva a una desarticulación de los distintos componentes con los resultados financieros; en otros términos, no conversa lo dicho cualitativamente con lo expresado en términos financieros.

Del mismo modo, y como ventajas asociadas al uso de la planeación financiera, se pueden destacar las siguientes:

- La planeación financiera es un proceso flexible y puede ser realizado en cualquier período de tiempo.

- Constituye una herramienta empresarial muy útil para evaluar los resultados financieros del negocio, al integrar todos los componentes del plan de negocio y traducirlos a términos financieros.
- Puede ser utilizada para fines internos (propios del proyecto) o externos, tales como negociaciones, inversiones, solicitud de créditos, toma de decisiones, entre otras.

- Los resultados de la planeación financiera están estrechamente vinculados con las estrategias y políticas adoptadas para el proyecto de creación de empresa.

- Es importante que para el proceso de modelación y proyección financiera, se tengan bien definidos y clarificados los parámetros o variables que impactan los resultados, tanto internas (de la empresa) como externas (factores macroeconómicos, políticas legales), ya que de estos dependen en gran medida los resultados de la proyección (García, 1999 \& Gutiérrez, 2007).

- La planeación financiera es una herramienta empresarial muy adaptable a cualquier tipo de proyecto de inversión, por lo que su buen uso permite fundamentar la creación de empresas sostenibles en el tiempo.

Adicionalmente, es conveniente hacer mención al carácter estratégico e integral que adquiere la planeación financiera en un proceso de creación de empresa, dado que se convierte en la base para la toma de decisiones acertadas, al permitir predecir los futuros comportamientos del negocio y sus repercusiones en la situación económica, contable y financiera del mismo (Varela 2001; Meléndez, 2005; Frixione, 2004 y Mejía 2005). En este sentido, podría decirse que la planeación financiera es el vehículo para la creación de empresas sostenibles, puesto que permite visualizar los resultados de las distintas áreas organizacionales en términos cuantitativos y de esta forma, intervenir en estos para que se logre un equilibrio económico en todos los niveles de la empresa, afrontando los retos y cambios que impone el entorno de la mejor forma.

Sólo a través de la planeación financiera, es posible materializar los efectos financieros de las decisiones estratégicas y operativas que se toman antes, durante y después de poner en marcha el proyecto de creación de empresa, por lo que es precisamente en este sentido que la planeación financiera adquiere real importancia como una herramienta de ges- 
tión empresarial que ayuda a mejorar los resultados financieros y decisiones que se tomen al interior de la organización, así como su repercusión en la creación de empresas sostenibles en el tiempo, con lo cual se podría hablar de una cultura de generación de valor en estas nuevas unidades económicas (Correa, Jaramillo, Ramírez \& Castaño, 2008).

\section{Falencias comunes (usuales) de los emprendedores al momento de realizar la planeación financiera}

Tal como se ha expresado, la planeación financiera permite cuantificar la situación futura de los proyectos empresariales y realizar una evaluación de sus resultados dependiendo de los estudios que se realizan en el plan de negocios. Sin embargo, cuando dicha planeación se realiza sin apoyo y de forma intuitiva, es posible cometer errores que repercutirán en el futuro del proyecto empresarial cuando realmente se ingrese al mercado y no se pueda sostener la empresa, dado que no se cuenta con los recursos suficientes para continuar. A continuación se presentan algunas falencias que se evidencian en los planes de negocio de muchos emprendedores que desconocen los beneficios de una planeación financiera adecuada para su proyecto.

\subsection{Falencia No. 1. Proyecciones de ingresos muy elevadas que no son acordes con los costos y gastos.}

Es posible evidenciar a través de los diversos planes de negocios, una tendencia en la cual los emprendedores realizan proyecciones bastante optimistas con relación a sus ingresos dado que confían demasiado en su potencial. No obstante, dichas consideraciones sobre ingresos no son tenidas en cuenta al estimar los costos y gastos que se derivan de dichas ventas futuras. A continuación, se presenta de forma gráfica tal situación en una proyección de un proyecto de creación de empresa real.

Como se puede observar en la ilustración 2, la tendencia de incrementos en los ingresos es bastante alta mientras que los costos y gastos permanecen en una línea casi constante. En este sentido, se hace evidente la necesidad de entrar a revisar cuáles son las causas que producen los fuertes aumentos de las ventas y por qué no producen un impacto significativo en los costos y gastos con el fin de mejorar las proyecciones financieras y lograr un resultado que se acerque a las posibilidades de la empresa que se creará a partir del plan de negocios.

\subsection{Falencia No. 2. Falta de presupuestos de capital para la adquisición de propiedad, planta y equipo teniendo en cuenta la obsolescencia de los activos iniciales.}

Es factible encontrar gran cantidad de proyectos de creación de empresas que presentan una clara inversión inicial en propiedad, planta y equipo. Sin embargo, teniendo en cuenta que la mayoría de proyecciones de este tipo de proyectos se realizan en rangos de 5 y 10 años, es común encontrar que no se realizarán inversiones en dichos activos para los siguientes años en los cuales la empresa ya estará en operación, dejando de esta forma que la empresa no se esté proyectando para su posterior crecimiento a través del incremento de sus activos fijos que además son necesarios para aumentar la capacidad productiva y acoger el nuevo personal que servirá a la organización. En la tabla No. 1 se puede observar la inversión en propiedad, planta y equipo de un proyecto de creación de empresa para un periodo de 5 años, tal como se describió.

La tabla 1, es una evidencia clara de un error de los emprendedores en la estimación de su propiedad, planta y equipo, ya que no tienen en cuenta la obsolescencia de sus activos, principalmente, de sus equipos de cómputo, para este caso particular. Además, no se tiene en cuenta que el negocio va a crecer y en este sentido, se debe incrementar la maquinaria y equipo para afrontar el aumento en la demanda de los bienes y servicios que ofrecerá la empresa.

\subsection{Falencia No. 3. Distribución de utilidades sin tener en cuenta el flujo de caja libre del proyecto (decisión basada sólo en la utilidad neta).}

Las decisiones sobre distribución de utilidades deben estar fundamentadas en diferentes aspectos 


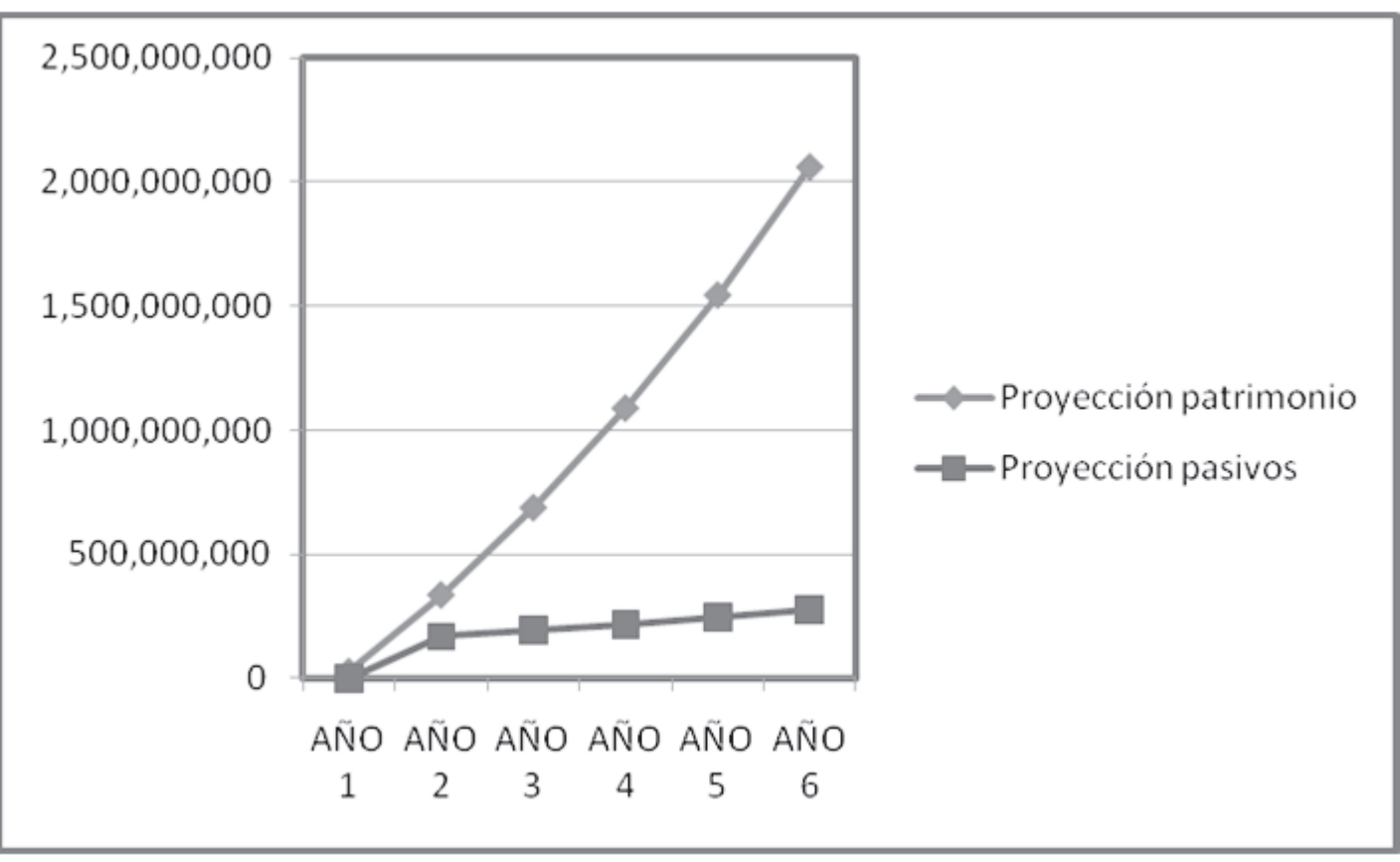

Ilustración 2. Proyección de ingresos vs. Proyecciones de costos y gastos²

Tabla 1. Proyección de las inversiones en propiedad, planta y equipo ${ }^{3}$

\begin{tabular}{|l|c|c|c|c|c|c|}
\hline \multicolumn{1}{|c|}{ INVERSIONES EN ACTIVOS FIJOS } & Inversión inicial & Año 1 & Año 2 & Año 3 & Año 4 & Año 5 \\
\hline Adecuación infraestructura & $\$ 30.000 .000$ & & & & & \\
\hline Total Maquinaria y Equipo: & $\$ 26.000 .000$ & $\$-$ & $\$-$ & $\$-$ & $\$-$ & $\$-$ \\
\hline Equipos 1 & $\$ 16.800 .000$ & & & & & \\
\hline Equipos 2 & $\$ 2.800 .000$ & & & & & \\
\hline Equipos 3 & $\$ 6.400 .000$ & & & & & \\
\hline Total Muebles y Enseres: & $\$ 2.930 .000$ & $\$-$ & $\$-$ & $\$-$ & $\$-$ & $\$-$ \\
\hline Mobiliario & $\$ 2.130 .000$ & & & & & \\
\hline Otros & $\$ 800.000$ & & & & & \\
\hline Total Equipo de Computo & $\$ 3.200 .000$ & & & & & \\
\hline Computadores & $\$ 3.200 .000$ & & & & & \\
\hline SUBTOTAL ACTIVOS FIJOS & $\$ 62.130 .000$ & $\$-$ & $\$-$ & $\$-$ & $\$-$ & $\$-$ \\
\hline
\end{tabular}

Elaboración de los autores a partir de la asesoría de varios planes de negocios. Elaboración de los autores a partir de la asesoría de varios planes de negocios. 
tales como: capitalización de la empresa, liquidez para el pago de los dividendos, inversión de las utilidades en proyectos de ensanchamiento de planta, fortalecimiento del patrimonio y de las finanzas de la empresa en general. En este sentido, se encuentra en muchos proyectos de creación de empresas la falta de asesoría para los emprendedores frente a un tema tan vital en las finanzas de una empresa como lo es la distribución de utilidades.

Desde la perspectiva planteada, es evidente que muchos emprendedores consideran que al obtener utilidades en el estado de resultados, dichas utilidades se convierten inmediatamente en recursos o beneficios a distribuir entre los socios o el socio único, sin tener en cuenta que dichas utilidades no se encuentran en términos de dinero en efectivo y que han sido afectados por partidas contables como la depreciación, amortización y provisión y que además, no considera el capital de trabajo y las variaciones brutas en los activos fijos operacionales.

En la tabla 2 se puede observar cómo los emprendedores toman decisiones de distribución de utilidades a partir de la utilidad neta y no a partir del flujo de caja del proyecto.

Tal como se puede observar en la tabla 2, en la proyección del año 1 la empresa obtendría una utilidad de $\$ 92.568 .984$, de la cual los emprendedores deciden distribuir como dividendos $\$ 37.027 .593$. No obstante, el flujo de caja libre operacional es negativo por un valor de \$(519.173.542) que se presentó dado que se piensa realizar en este proyecto una gran inversión en activos fijos para el año 1 . De esta forma, es claro que para distribuir dividendos, la empresa tendría que endeudarse para cubrir las adquisiciones de activos fijos y también para pagar dichos dividendos. Para el año 2, es claro que aunque la empresa obtiene flujos de caja positivos por valor de $\$ 20.726 .389$, no será posible pagar con recursos propios los dividendos que ascienden a una suma de \$24.205.974, por lo tanto, se tendrá que recurrir nuevamente al endeudamiento para cubrir el pago a los socios.

La situación que se presenta en esta temática hace necesaria una intervención por parte de los asesores de los planes de negocio para que instruyan a los emprendedores en un tema tan crucial como lo es la comprensión de la diferencia entre los flujos de caja libre y las utilidades que se encuentran en el estado de resultados.

\subsection{Falencia No. 4. Crecimientos en ventas que superan la capacidad instalada sin planes de ampliación en planta}

Por lo general, muchos de los emprendedores plantean unas ventas para el primer año que ocupan para los últimos meses entre el $80 \%$ y el $100 \%$ de su capacidad de producción instalada con el fin de mostrar unos resultados bastante buenos desde el primer año que les permita presentar un proyecto auto-sostenible y con una rentabilidad atractiva para los inversionistas. Sin embargo, al realizar la para-

Tabla 2. Proyecciones de utilidad neta y flujo de caja libre operacional ${ }^{4}$

\begin{tabular}{|l|c|c|l|c|c|}
\hline & Año 1 & Año 2 & & Año 1 & Año 2 \\
\hline Utilidad/Pérdida Neta & $\$ 92.568 .984$ & $\$ 60.514 .935$ & $\begin{array}{l}\text { Flujo de caja libre ope- } \\
\text { racional }\end{array}$ & $\$(519.173 .542)$ & \$20.726.389 \\
\hline Reserva legal & $\$ 9.256 .898$ & $\$ 6.051 .494$ & & & \\
\hline Utilidades acumuladas & $\$ 46.284 .492$ & $\$ 30.257 .468$ & & \\
\hline Dividendos & $\$ 37.027 .593$ & $\$ 24.205 .974$ & & \\
\hline
\end{tabular}

4 Elaboración de los autores a partir de la asesoría de varios planes de negocios. 
metrización para la proyección de ventas parece que olvidaran los limitantes de su capacidad instalada en planta y simplemente plantean incrementos en ventas que van ligados al aumento de la producción para cada uno de los siguientes años.

Es de notar que después de observar las ventas frente a la capacidad instalada del proyecto empresarial se podrá evidenciar la necesidad de incrementar tanto la propiedad, planta y equipo como el personal que labora en la empresa, así como algunos costos y gastos que requerirán mayor inversión frente a los aumentos de la productividad. De este modo, quienes desarrollan el plan de negocios tendrán que pensar en el impacto que producen sus decisiones en cualquiera de los componentes ya que como se ha mencionado todos impactan al componente financiero.

Por otro lado, una suficiente administración de los recursos en el proyecto empresarial permitirá que el o los emprendedores cuenten con una verdadera herramienta de decisión hacia el futuro: la planeación financiera, la cual permitirá guiar a la empresa hacia el cumplimiento de las metas empresariales y el denominado objetivo básico financiero.

\subsection{Falencia No. 5. Pérdidas iniciales que generan causal de disolución por su representatividad frente al capital}

En algunos casos, las proyecciones de estados financieros de los proyectos de creación de empresa arrojan resultados negativos en los primeros años. Dichas pérdidas que se presentan, superan en muchas ocasiones el capital aportado o el patrimonio neto generando de esta forma una causal de disolución especial dependiendo del tipo de régimen jurídico que se acoja. A continuación se presentan las reglas especiales para los casos de disolución que plantea el Código de Comercio colombiano:

- Para la sociedad anónima se plantea que: "cuando se incurran pérdidas que reduzcan el patrimonio neto por debajo del cincuenta por ciento del capital suscrito" (art. 457).

- Sociedad en comandita simple "por pérdida que reduzca su capital a la tercera parte o menos" (art. 342).

- Sociedad en comandita por acciones "cuando ocurran pérdidas que reduzcan el patrimonio neto a menos del cincuenta por ciento del capital suscrito" (art. 351).

- Sociedad de responsabilidad limitada "cuando ocurran pérdidas que reduzcan el capital por debajo del cincuenta por ciento" (art. 370).

- Para la sociedad por acciones simplificada se tiene que se estará en causal de disolución cuando se incurra en "pérdidas que reduzcan el capital suscrito por debajo del 50\% del capital suscrito" (art. 34 de la ley 1258 de 2008).

De este modo, los emprendedores deben ser conscientes de las situaciones planteadas y tener en cuenta que al incurrir en pérdidas para los primeros años que generan causal de disolución deben replantear sus estudios técnicos y de mercadeo con el fin de subsanar dichos problemas que se tendrían en el futuro. En la tabla 3 se puede observar un ejemplo de un proyecto, el cual desean constituir como una Sociedad por Acciones Simplificada (S.A.S.).

Tabla 3. Proyección del patrimonio ${ }^{1}$

\begin{tabular}{|l|r|r|r|r|}
\hline \multicolumn{1}{|c|}{ PATRIMONIO } & \multicolumn{1}{c|}{ Inicial } & AÑO 1 & AÑO 2 & AÑO 3 \\
\hline Capital & 13.200 .000 & 13.200 .000 & 13.200 .000 & 13.200 .000 \\
\hline Reservas & 0 & 0 & 0 & 0 \\
\hline Utilidad del Periodo & 0 & -33.466 .437 & -6.278 .213 & 17.838 .754 \\
\hline Utilidad o pérdida de Ejercicios Anteriores & 0 & 0 & -33.466 .437 & -39.744 .650 \\
\hline TOTAL PATRIMONIO & 13.200 .000 & -20.266 .437 & -26.544 .650 & -8.705 .895 \\
\hline
\end{tabular}

5 Elaboración de los autores a partir de la asesoría de varios planes de negocios. 
Se observa que para el primer año se incurrirá en pérdidas que reducen el capital suscrito a menos del $50 \%$ y por lo tanto se incurre en causal de disolución. Es por ello, que los emprendedores de este proyecto tendrán que replantear su situación para el primer año u observar estrategias que le permitan continuar con su proyecto empresarial, por ejemplo, subsanando la situación en un período inferior a 18 meses tal como lo expresa la ley 1258 de 2008.

\subsection{Falencia No. 6. Deficiente estimación de los costos.}

En cuanto al tema de los costos, los emprendedores presentan diversas dificultades para realizar la estimación de cada uno de los componentes del costo que hacen parte de su producto o servicio. Es por ello, que en muchas situaciones se encuentran proyecciones de costos que no tienen en cuenta muchos de los elementos del costo o parte de ellos. Ejemplos de esta situación son: no realizar cotizaciones y estimar los costos de forma intuitiva, olvidar las prestaciones sociales y las obligaciones de seguridad social para la mano de obra directa, no tener en cuenta la depreciación de la propiedad, planta y equipo, no incluir materiales de empaque del producto, calcular de forma deficiente el valor de materia prima por unidad, entre otros. Dado lo anterior, el estudio técnico tendrá que ser más preciso con el fin de obtener una buena estimación de costos y realizar proyecciones que se acerquen a la realidad futura de la empresa y que permitan su continuidad ya que los costos son un aspecto que, entre otros, le permitirá a la organización ser más competitiva en el mercado.

\subsection{Falencia No. 7. El endeudamiento en el largo plazo pierde importancia y en algunos casos tiende a "cero".}

Frente a los procesos de planeación financiera de los planes de negocio, se encuentra que diversos emprendedores utilizan el endeudamiento como herramienta para el inicio de sus operaciones. No obstante, estos pasivos (principalmente obligaciones financieras) son canceladas en el horizonte de evaluación del proyecto sin mayor explicación que la de unos flujos de caja bastante optimistas que permiten el pago de dichas obligaciones, con lo cual, se producen tendencias del endeudamiento hacia "cero". En la ilustración 3, se puede observar como una proyección patrimonial, supera en gran medida al crecimiento de los pasivos que se generan básicamente por los impuestos por pagar que se derivan del cierre de fin de año en las organizaciones o posiblemente, a través de la parametrización del endeudamiento con proveedores que depende directamente del costo de producción o prestación de servicios.

Adicional a la situación anterior, la ilustración 4 permite observar como el nivel de endeudamiento total (total pasivo/total activo), desciende vertiginosamente a través del horizonte de evaluación del proyecto de creación de empresa. En este caso, la nueva empresa iniciaría con un fuerte nivel de endeudamiento que supera el 100\%, para llegar en el año 5 a un nivel de endeudamiento aproximado al $20 \%$. Con esto, el emprendedor olvida que es posible que su empresa en los siguientes años pueda seguir recurriendo a la financiación como mecanismo de apalancamiento para lograr mayores crecimientos tanto a nivel operativo como financiero. Además, el endeudamiento permite darle mayor solidez a la futura empresa, ya que facilita la adquisición de nuevos activos, el crecimiento en planta y capacidad operativa, la apertura de agencias y sucursales, la inversión en tecnología y desarrollo, entre otros.

En términos generales, las anteriores falencias, que se evidencian a partir del acompañamiento a varios planes de negocio, no pretenden agotar las falencias que se pueden encontrar a nivel financiero en los planes de negocio, sino que se pusieron de manifiesto a fin de llamar la atención sobre algunos errores comunes en que usualmente se incurre en la formulación financiera de proyectos, de tal forma que emprendedores y consultores discutan con mayor profundidad los planteamientos financieros que realizan con el fin de crear empresas sostenibles en el tiempo. 


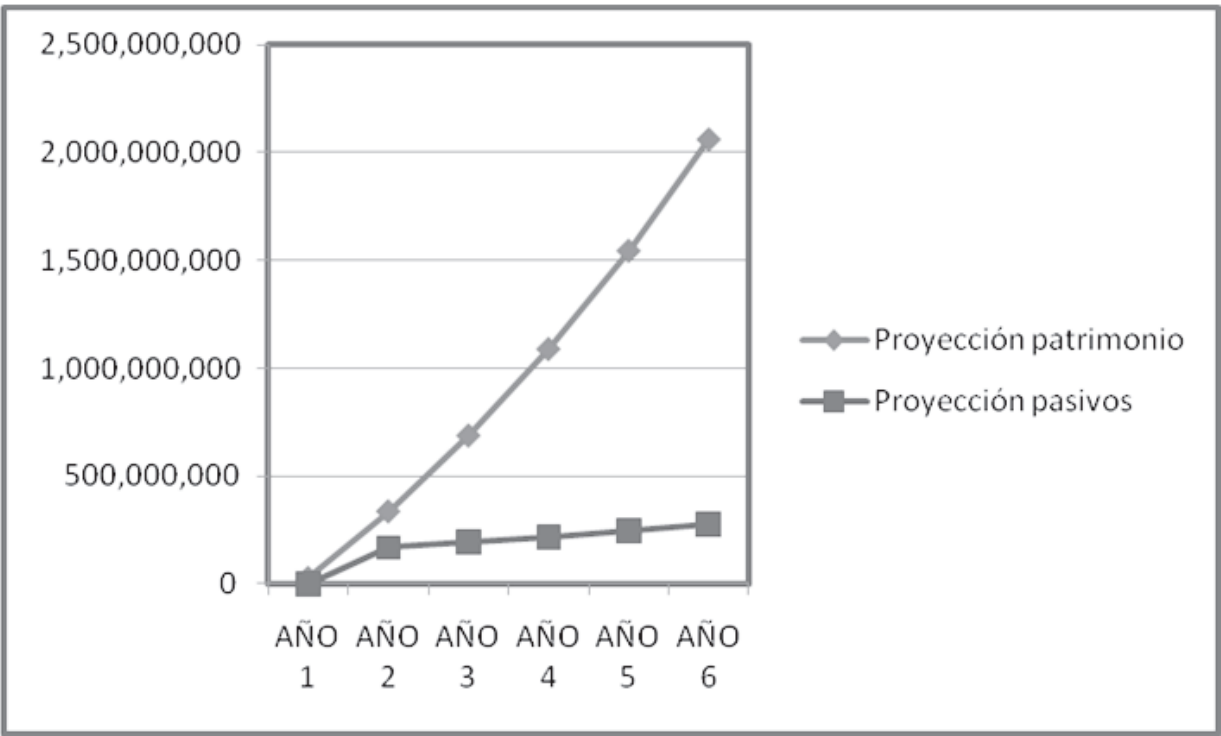

Ilustración 3. Proyección del patrimonio vs. Proyección de pasivos ${ }^{6}$

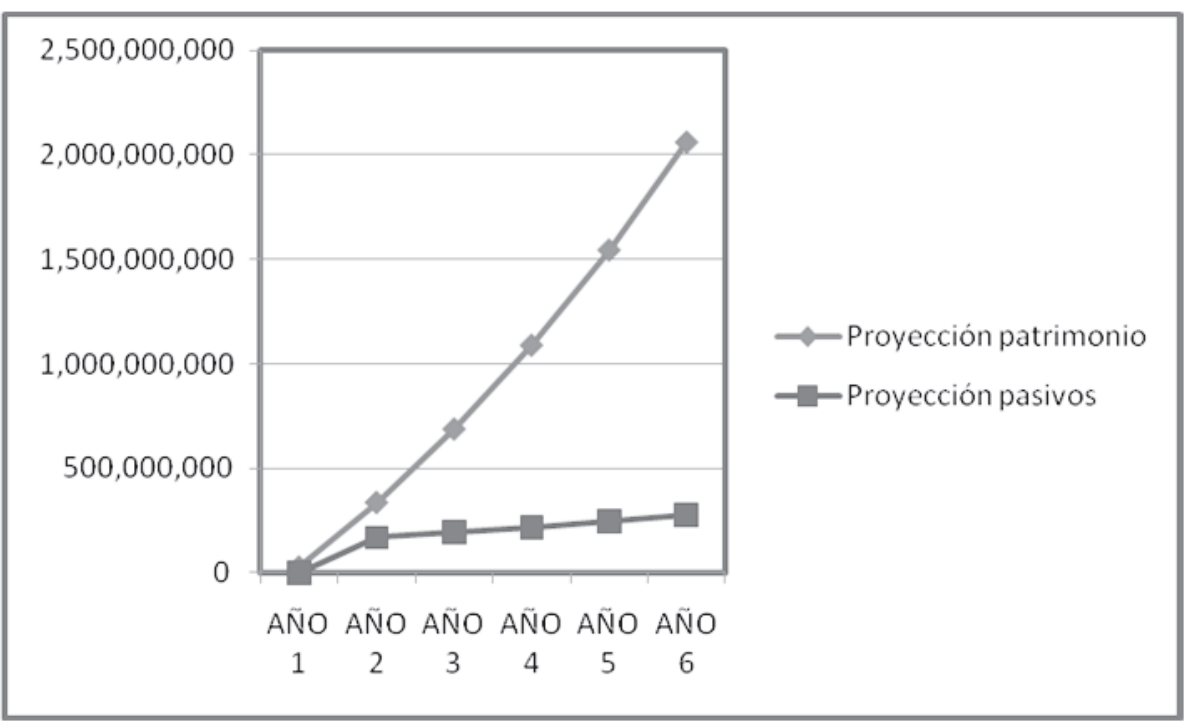

Ilustración 4. Proyección endeudamiento.

\footnotetext{
Elaboración de los autores a partir de la asesoría de varios planes de negocios. Elaboración de los autores a partir de la asesoría de varios planes de negocios.
} 


\section{Consideraciones finales}

La falta de formulación de un plan de negocios dificulta la puesta en marcha del mismo, por lo que su supervivencia y posterior crecimiento y desarrollo se ven afectados. Es importante realizar una evaluación previa, algo así como un diagnóstico, a fin de conocer las condiciones y situaciones a las que está y estará sujeta la empresa, y de esta manera poder cuantificar y controlar los posibles riesgos asociados a la operación normal de ésta (Varela, 2001, Correa \& Jaramillo, 2007).

Por lo anterior, la elaboración de planes de negocios sustentados en la planeación financiera constituye el fundamento ideal para la creación de empresas sostenibles en el tiempo. De ahí que el carácter estratégico de la planeación financiera, como motor que impulsa la creación de empresas sostenibles, sea la base principal para su consolidación y expansión (Castaño, 2009). Asimismo, la planeación financiera constituye una herramienta de gestión empresarial sumamente importante que permite visualizar el proyecto de creación de empresa de forma global en términos financieros, por lo que las estrategias y planes operativos pueden ser cuantificados y proyectados a futuro.

Por otro lado, muchos de los procesos actuales de planeación financiera han traído consigo algunos errores que se evidencian en el módulo financiero de los planes de negocio elaborados por los emprendedores. De este modo, la carencia de capacitación en el tema financiero para los emprendedores hace que éstos, no encuentren en el proceso de planeación financiera un apoyo estratégico a la hora de crear su empresa y gestionarla con suficiencia posteriormente.

Por último, se logra identificar a través de este texto la importancia de la planeación financiera en la elaboración de planes de negocios para la creación de empresas que sean sostenibles en el tiempo. Asimismo, se abre la puerta para la investigación sobre estas dificultades financieras que se pueden evidenciar a través de la evaluación de los planes de negocio de los futuros empresarios.

\section{Referencias}

Black, L. \& Tarkin, A. (2006). Ingeniería económica (6ª ed.). México: Mc Graw Hill.

Castaño, C. E. (2009). El carácter estratégico de la contabilidad en las MIPYMES: el contexto colombiano. Disponible en: http://contaduria.udea.edu.co/proyecto/archivos/rev/rev32/ El\%20caracter\%20estrategico\%20de\%20la\%20contabilidaCastano.pdf. Recuperado el 8 de enero de 2010.

Castaño, C. E. \& Ramírez, L. J. (2009). Contabilidad para MIPYMES en Colombia: "Contexto y estrategia". En: Revista Gestión Jóven. Disponible en: http://www.elcriterio.com/revista/ajoica/contenidos_3/contabilidad_para_mipymes.pdf. Recuperado el 15 de enero de 2010).

Código de Comercio de Colombia.

Correa, J. A., Jaramillo, F., Ramírez, L. J. \& Castaño, C. E. (2008). ¿Es factible en las PYMES la valoración y la creación de valor? En: Lúmina, Universidad de Manizales, (9): 20-46.

Correa, J. A. \& Jaramillo, F. (2007). Una aproximación metodológica y prospectiva a la gestión financiera en las pequeñas empresas. En: Contaduría Universidad de Antioquia, (50): 93-118.

Frixione, D. (2004). Autodiagnóstico de pequeñas y medianas empresas. México: Limusa.

García, O. L. (1999). Administración financiera: fundamentos y aplicaciones ( $3^{a}$ ed.). Cali: prensa moderna impresores.

Gutiérrez, J. (2007). Modelos financieros con Excel: herramientas para mejorar la toma de decisiones empresariales. Bogotá: ECOE ediciones.

Mejía, T. A. (2005). Estructura presupuestal de un proyecto económico. Bogotá: Universidad Santo Tomás.

Meléndez, H. (2005). Plan de negocios y análisis de inversiones. Bucaramanga: Universidad Santo Tomás.

Ministerio de Comercio, Industria y Turismo -MCIT de Colombia. (2006). Plan de negocios Disponible en: http://www.mipymes.gov.co/pyme/verimp. asp?id=154\&idcompany $=43$. Recuperado el 28 de Mayo de 2009.

Programa Gestión Tecnológica, Universidad de Antioquia. (2008). Plan de Negocios. Disponible en: http://gestiontecnologica. udea.edu.co/index. php? Itemid $=55 \&$ id $=21 \&$ option $=$ com content\&task=view. Recuperado el 28 de mayo de 2009

Ramírez, H. F. \& Vanegas, B. (2008). Gestión de costos en las PYMES: problemática y alternativas de solución. Bogotá: Universidad Libre de Colombia.

Ramírez, J. C. (2006). Pymes más competitivas (2a ed). Bogotá: ediciones Mayol.

Sánchez, A. (2006). La planeación financiera: soporte para decisiones estratégicas. En: Revista MM, edición especial, (50): 154-159.

Sapag, N. \& Sapag, R. (2003). Preparación y evaluación de proyectos (4a ed.). México: Mc Graw Hill.

Soto, E. \& Dolan, S. L. (2004). Las Pymes ante el reto del siglo XXI: Ios nuevos mercados globales. México: Thomson.

Varela, R. (2001). Innovación empresarial: arte y ciencia en la creación de empresas ( $2^{a}$ ed.). Bogotá: Pearson educación de Colombia. 